\title{
Global well-posedness of compressible magnetohydrodynamics with density-dependent viscosity and resistivity
}

\author{
Menglong Sư ${ }^{1 *}$, Yingzhao Liu', Wenzhuang Zhu ${ }^{2}$ and Jian Wang ${ }^{3}$
}

"Correspondence:

mlsulynu@163.com

${ }^{1}$ School of Mathematical Sciences,

Luoyang Normal University,

Luoyang, 471022, P.R. China

Full list of author information is

available at the end of the article

\begin{abstract}
In this paper, we study the initial-boundary value problem for one-dimensional compressible magnetohydrodynamics (MHD) flows. Using the local estimates of strong solutions to three-dimensional compressible MHD (obtained by Fan and Yu in Nonlinear Anal. 69(10):3637-3660, 2008) and Sobolev's inequalities, we get the unique global classical solution $(\rho, u, b)$, where $\rho \in C^{1}\left([0, T] ; H^{1}([0,1])\right), u \in H^{1}\left([0, T] ; H^{1}([0,1])\right)$, and $b \in C^{1}\left([0, T] ; H^{1}([0,1])\right)$ for any $T>0$. Here, we emphasize that the initial density $\rho_{0}$ is permitted to contain vacuum states and the initial velocity $u_{0}$ and the magnetic field $b_{0}$ can be arbitrarily large. Also, both the viscosity coefficient $\mu$ and the resistivity coefficient $v$ depend on the density $\rho$.
\end{abstract}

Keywords: global classical solutions; compressible magnetohydrodynamics flows; vacuum states

\section{Introduction}

The mathematical model of magnetohydrodynamics (MHD) is used to simulate the motion of a conducting fluid under the effect of the electromagnetic field and has a very wide range of applications in astrophysics, plasma, and so on. The governing equations of MHD can be stated as follows $(c f .[1,2])$ :

$$
\left\{\begin{array}{l}
\rho_{t}+(\rho u)_{x}=0 \\
(\rho u)_{t}+\left(\rho u^{2}\right)_{x}+P_{x}(\rho)+\frac{1}{2}\left(b^{2}\right)_{x}=\left(\mu(\rho) u_{x}\right)_{x} \\
b_{t}+(b u)_{x}=\left(v(\rho) b_{x}\right)_{x}
\end{array}\right.
$$

associated with the initial and boundary conditions:

$$
\left\{\begin{array}{l}
(\rho, u, b)(0, x)=\left(\rho_{0}, u_{0}, b_{0}\right)(x), \quad \text { for } x \in[0,1] \\
\left.u\right|_{x=0,1}=\left.b\right|_{x=0,1}=0, \quad \text { for } t \geq 0
\end{array}\right.
$$

The unknown functions $\rho, u, P(\rho)$, and $b$ denote the fluid density, velocity, pressure, and magnetic field, respectively. The assumptions on the viscosity coefficient $\mu(\rho)$ and the resistivity coefficient $v(\rho)$ depend on the density $\rho$, which is mainly due to the physical meaning (cf. [3]). For simplicity, we only consider the polytropic gas, i.e., $P=P(\rho)=a \rho^{\gamma}$ with $a>0$ and $\gamma>1$ being constants.

c) 2015 Su et al: licensee Springer. This is an Open Access article distributed under the terms of the Creative Commons Attribution License (http://creativecommons.org/licenses/by/4.0), which permits unrestricted use, distribution, and reproduction in any medium, provided the original work is properly credited. 
In this paper, we will focus on the existence of the global classical solutions to the initialboundary value problem (1)-(2). Before we present our main result, we first recall some of the previous results concerning the compressible MHD. Lots of work has been done on the global existence and the regularity of the solutions, we begin with the one-dimensional case. The existence and uniqueness of local smooth solutions were proved firstly in [4], while the existence of global smooth solutions with small smooth initial data was shown in [5]. The exponential stability of small smooth solutions was obtained in [6, 7]. Recently, Fan et al. [8, 9] obtained the existence, the uniqueness and the Lipschitz continuous dependence on the initial data of global weak solutions of compressible MHD when the initial data lie in the Lebesgue spaces. In addition, Fan et al. [10] obtained the global strong solutions to the planar compressible MHD with large initial data and vacuum.

For the multi-dimensional compressible MHD equations, there are also lots of mathematical results. Volpert and Hudjaev [4] first obtained the local smooth solutions to the compressible MHD equations as mentioned before. Li et al. [11] obtained the existence and uniqueness of local strong solutions in time ith large initial data when the initial density has a positive lower bound. Fan and $\mathrm{Yu}$ [12] obtained the strong solutions to the compressible MHD equations with vacuum. Kawashima [13] obtained the smooth solutions for two-dimensional compressible MHD equations when the initial data is a small perturbation of a given constant state. Umeda et al. [14] obtained the decay of solutions to the linearized MHD equations. Li and $\mathrm{Yu}$ [15] obtained the optimal decay rate of small smooth solutions. In [16, 17], Hu and Wang obtained the global existence of weak solutions to the isentropic compressible MHD equations and variational solutions to the full compressible MHD equations; see also [18-20] for related results. Suen and Hoff [21] obtained the global low-energy weak solutions of the isentropic compressible MHD equations. Later, Liu et al. [22] obtained the global weak solution with discontinuous initial data when the initial energy is small enough. Under the assumption that the initial energy is sufficiently small, Li et al. [23] obtained the large time existence of classical solutions to the compressible MHD which may have large oscillations and vacuum. At the same time, they also obtained the large time behavior as follows:

$$
\lim _{t \rightarrow \infty}\left(\|\rho(\cdot, t)-\tilde{\rho}\|_{L^{p}}+\int \rho^{1 / 2}|u|^{4} d x+\|\nabla u(\cdot, t)\|_{L^{r}}+\|\nabla b(\cdot, t)\|_{L^{r}}\right)=0,
$$

for $r \in[2,6)$ and

$$
p \in \begin{cases}(\gamma, \infty), & \text { if } \tilde{\rho}=0 \\ (2, \infty), & \text { if } \tilde{\rho}>0\end{cases}
$$

The large time behavior was recently improved by Lv et al. in [24], precisely speaking,

$$
\begin{cases}\|\nabla b\|_{L^{p}} \leq C t^{-1+(6-p) / 4 p}, & \text { for } p \in[2,6], \\ \|\nabla u(\cdot, t)\|_{L^{p}} \leq C t^{-1+1 / p}, & \text { for } p \in[2,6], \\ \|P(\cdot, t)\|_{L^{r}} \leq C(r) t^{-1+1 / r}, & \text { for } r \in(1, \infty)\end{cases}
$$

where $C(r)$ and $C$ both depend on $\left\|\rho_{0}\right\|_{L^{1}\left(\mathbb{R}^{3}\right)}$ as $\gamma>3 / 2$.

Here we point out that although there are many progress on compressible MHD equations, it is still an open question to obtain the global strong or smooth solutions to the 
full compressible MHD equations with large initial data and possible vacuum even in the one-dimensional case; see [17].

To proceed, we first introduce the notions and conventions used throughout the paper. We denote

$$
\int f d x=\int_{I} f d x
$$

where $I=(0,1)$ is the space interval. For $p \geq 1, L^{p}=L^{p}(I)$ denotes the $L^{p}$ space with the norm $\|\cdot\|_{L^{p}}$. For $k \geq 1$ and $p \geq 1, W^{k, p}=W^{k, p}(I)$ denotes the Sobolev space, whose norm is denoted $\|\cdot\|_{W^{k, p}}$, and $H^{k}=W^{k, 2}$. For $k \geq 0$ and $0<\alpha<1$, let $C^{k+\alpha}$ denote the Schauder function space on $I$, whose $k$ th order derivative is Hölder continuous with the exponent $\alpha$ and with the norm $\|\cdot\|_{C^{k+\alpha}}$.

Our main result is stated as follows.

Theorem 1.1 Assume that $\rho_{0} \geq 0, \rho_{0} \in H^{2}, \rho_{0}^{\gamma} \in H^{2},\left(u_{0}, b_{0}\right) \in H^{3} \cap H_{0}^{1}$, and the initial data satisfy the following compatibility conditions:

$$
\left[\mu\left(\rho_{0}\right) u_{0 x}\right]_{x}(x)-\left[P\left(\rho_{0}\right)\right]_{x}(x)=\rho_{0}(x) g(x), \quad x \in[0,1],
$$

for a given function $g \in H_{0}^{1}$. Furthermore, assume that the viscosity and the resistivity coefficient satisfy

$$
\mu \in C^{2}[0, \infty), \quad 0<M_{1} \leq \mu(\rho) \leq M_{2}\left(1+\rho^{\gamma}\right), \quad \text { for any } \rho \geq 0,
$$

and

$$
v \in C^{2}[0, \infty), \quad 0<N \leq v(\rho), \quad \text { for any } \rho \geq 0,
$$

where $M_{1}, M_{2}$, and $N$ are some positive constants.

Then for any $T>0$ there exists a unique global classical solution $(\rho, u, b)$ to the initialboundary value problem (1)-(2) satisfying

$$
\begin{aligned}
& \left(\rho, \rho^{\gamma}\right) \in C\left([0, T] ; H^{2}\right), \quad\left(\rho_{t},\left(\rho^{\gamma}\right)_{t}\right) \in C\left([0, T] ; H^{1}\right), \\
& \rho_{t t} \in C\left([0, T] ; L^{2}\right), \quad \rho \geq 0, \quad\left(\rho^{\gamma}\right)_{t t} \in L^{\infty}\left([0, T] ; L^{2}\right), \\
& (\rho u)_{t} \in C\left([0, T] ; H^{1}\right), \quad(u, b) \in C\left([0, T] ; H^{3} \cap H_{0}^{1}\right), \\
& u_{t} \in L^{\infty}\left([0, T] ; H_{0}^{1}\right) \cap L^{2}\left([0, T] ; H^{2}\right), \\
& b_{t} \in C\left([0, T] ; H^{1}\right), \quad b_{t t} \in C\left([0, T] ; L^{2}\right) .
\end{aligned}
$$

Remark 1.1 For the assumption (3) on the initial data, which we called compatibility condition, was first introduced in [25] to study the viscous compressible fluid. After that, Kim et al. studied the local well-posedness of compressible fluid in a series papers ( $c f$. [26-28]). Roughly speaking, the compatibility condition (3) is equivalent to the $L^{2}$-integrability of $\sqrt{\rho} u_{t}$ at $t=0$, which is natural and plays a crucial role in deducing the regularity of the time derivatives of $u$. 
Remark 1.2 For mathematical technique, we assume that the viscosity $\mu(\rho)$ and the resistivity coefficient $\nu(\rho)$ satisfy (4) and (5), respectively. Precisely, the lower bound of $\mu(\rho)$ and $v(\rho)$ will be used to improve the regularity of the velocity $u$ and the magnetic fields $b$, respectively. The upper bound of $\mu(\rho)$ will be used to deduce the upper bound of the density $\rho$, which plays a crucial role in the analysis of the classical solution of the compressible MHD.

Remark 1.3 In Theorem 1.1, because $\rho_{0} \in H^{2}$ cannot imply $\rho_{0}^{\gamma} \in H^{2}$ with $\gamma \in(1,2)$, we assume that $\rho_{0}^{\gamma} \in H^{2}$ as well as $\rho_{0} \in H^{2}$.

The rest of the paper is organized as follows. In Section 2, we prove Theorem 1.1 by giving the initial density with a lower bound $\delta>0$, getting a sequence of approximate solutions to (1)-(2) and taking $\delta \rightarrow 0^{+}$after making some uniform estimates for $\delta$ on the approximate solutions.

\section{Proof of Theorem 1.1}

This section we devote to proving Theorem 1.1. Since the proof of local existence and uniqueness of strong solutions to the approximate problem is now standard in [12], thus we only focus on a priori estimates of the solutions to the initial-boundary value problem (1)-(2). For any given $T \in(0, \infty)$, let $(\rho, u, b)$ be the classical solution to (1)-(2). Then we have the following basic energy estimate.

Lemma 2.1 For any $0 \leq t \leq T$, one obtains that

$$
\sup _{0 \leq t \leq T} \int\left(\rho u^{2}+b^{2}+\rho^{\gamma}\right) d x+\int_{0}^{T} \int\left(u_{x}^{2}+b_{x}^{2}\right) d x d t \leq C,
$$

where $C$ is some generic constant depending on the initial data, the viscosity and electrical resistivity, and may change line by line.

Proof Multiplying the second and third equations in (1) by $u$ and $b$, integrating the resulting equations over $I$ and summing them together, then using integration by parts and from the conditions (4) and (5), we can show that (6) holds. This completes the proof.

Lemma 2.2 For any $(s, y) \in Q_{T}$, we have

$$
0 \leq \rho(s, y) \leq C .
$$

Proof Denote

$$
w(t, x)=\int_{0}^{t}\left[\mu(\rho) u_{x}-\rho u^{2}-\rho^{\gamma}-\frac{1}{2} b^{2}\right](s, x) d s+\int_{0}^{x}\left(\rho_{0} u_{0}\right)(y) d y .
$$

Differentiating (8) with respect to $x$ and using the second equation in (1), we have

$$
w_{x}=\rho u,
$$


which together with (6) and Hölder's inequality yields

$$
\int\left|w_{x}\right| d x \leq C
$$

From (4), (6), and (8), we conclude

$$
\int|w| d x \leq C
$$

Due to $W^{1,1} \hookrightarrow L^{\infty}$, one obtains

$$
\|w\|_{L^{\infty}\left(Q_{T}\right)} \leq C .
$$

For any $(s, y) \in Q_{T}$, let $x(t, y)$ satisfy

$$
\left\{\begin{array}{l}
\frac{d x(t, y)}{d t}=u(t, x(t, y)), \quad 0 \leq t<s \\
x(s, y)=y
\end{array}\right.
$$

Denote

$$
F(t, x)=\exp \left\{\int_{1}^{\rho(t, x)} \frac{\mu(\xi)}{\xi} d \xi+w(t, x)\right\} .
$$

It is easy to verify

$$
\begin{aligned}
\frac{d F(t, x(t, y))}{d t} & =\frac{\partial F}{\partial t}+u \frac{\partial F}{\partial x} \\
& =F\left(\frac{\mu(\rho)}{\rho} \rho_{t}+w_{t}+\frac{\mu(\rho)}{\rho} \rho_{x} u+\rho u^{2}\right) \\
& =-\left(\rho^{\gamma}+\frac{1}{2} b^{2}\right) F,
\end{aligned}
$$

which together with the definition of $F(t, x)$ yields

$$
\frac{d F(t, x(t, y))}{d t} \leq 0 .
$$

Integrating (11) with respect to $t$ over $(0, s)$ and using (4), one obtains

$$
F(s, y) \leq F(0, x(0, y)) \leq C .
$$

By the above inequality and (9), we obtain

$$
\exp \left\{\int_{1}^{\rho(s, y)} \frac{\mu(\xi)}{\xi} d \xi\right\} \leq \exp \{-w(s, y)\} \leq C,
$$

which together with (4) and (6) yields

$$
\rho(s, y) \leq C .
$$


From (4), (9), and (11), it is easy to obtain

$$
\rho(s, y) \geq 0 \text {. }
$$

Therefore, we complete the proof of Lemma 2.2.

Lemma 2.3 plays a key role in the proof of Theorem 1.1 .

Lemma 2.3 For any $0 \leq t \leq T$, one obtains

$$
\sup _{0 \leq t \leq T} \int\left(u_{x}^{2}+b_{x}^{2}\right) d x+\int_{0}^{T} \int\left(\rho u_{t}^{2}+b_{t}^{2}\right) d x d t \leq C .
$$

Proof Using the first equation in (1), we rewrite the second equation in (1) as

$$
\rho u_{t}+\rho u u_{x}+\left(\rho^{\gamma}\right)_{x}+\frac{1}{2}\left(b^{2}\right)_{x}=\left(\mu(\rho) u_{x}\right)_{x}
$$

Multiplying (13) by $u_{t}$, integrating the resulting equation over $I$ with respect to $x$, using integration by parts and from Cauchy's inequality, we conclude

$$
\begin{aligned}
\int \rho u_{t}^{2} d x+\frac{1}{2} \frac{d}{d t} \int \mu(\rho) u_{x}^{2} d x \\
=\frac{d}{d t} \int \rho^{\gamma} u_{x} d x+\frac{1}{2} \frac{d}{d t} \int b^{2} u_{x} d x-\int b b_{t} u_{x} d x-\gamma \int \rho^{\gamma-1} \rho_{t} u_{x} d x \\
\quad+\frac{1}{2} \int \mu^{\prime}(\rho) \rho_{t} u_{x}^{2} d x-\int \rho u u_{x} u_{t} d x \\
\leq \frac{d}{d t} \int \rho^{\gamma} u_{x} d x+\frac{1}{2} \frac{d}{d t} \int b^{2} u_{x} d x+\frac{1}{4} \int b_{t}^{2} d x+C \int b^{2} u_{x}^{2} d x \\
\quad+\gamma \int \rho^{\gamma-1}(\rho u)_{x} u_{x} d x-\frac{1}{2} \int \mu^{\prime}(\rho)(\rho u)_{x} u_{x}^{2} d x \\
\quad+\frac{1}{2} \int \rho u_{t}^{2} d x+C \int \rho u^{2} u_{x}^{2} d x .
\end{aligned}
$$

Multiplying the third equation in (1) by $b_{t}$, integrating the resulting equation with respect to $x$ over $I$, using integration by parts and from Cauchy's inequality, we obtain

$$
\begin{aligned}
& \int b_{t}^{2} d x+\frac{1}{2} \frac{d}{d t} \int v(\rho) b_{x}^{2} d x \\
& =\frac{1}{2} \int v^{\prime}(\rho) \rho_{t} b_{x}^{2} d x-\int b_{x} u b_{t} d x-\int b u_{x} b_{t} d x \\
& \leq-\frac{1}{2} \int v^{\prime}(\rho)(\rho u)_{x} b_{x}^{2} d x+\frac{1}{4} \int b_{t}^{2} d x+C \int\left(b_{x}^{2} u^{2}+b^{2} u_{x}^{2}\right) d x
\end{aligned}
$$

Combining (14) and (15), we obtain

$$
\begin{aligned}
& \int\left(\rho u_{t}^{2}+b_{t}^{2}\right) d x+\frac{d}{d t} \int\left[\mu(\rho) u_{x}^{2}+v(\rho) b_{x}^{2}\right] d x \\
& \leq 2 \frac{d}{d t} \int \rho^{\gamma} u_{x} d x+\frac{d}{d t} \int b^{2} u_{x} d x+C \int\left(b^{2} u_{x}^{2}+\rho u^{2} u_{x}^{2}+b_{x}^{2} u^{2}\right) d x
\end{aligned}
$$




$$
\begin{aligned}
& +2 \gamma \int \rho^{\gamma-1}(\rho u)_{x} u_{x} d x-\int \mu^{\prime}(\rho)(\rho u)_{x} u_{x}^{2} d x-\int v^{\prime}(\rho)(\rho u)_{x} b_{x}^{2} d x \\
& \leq 2 \frac{d}{d t} \int \rho^{\gamma} u_{x} d x+\frac{d}{d t} \int b^{2} u_{x} d x \\
& +C\left[\left(\int v(\rho) b_{x}^{2} d x\right)\left(\int \mu(\rho) u_{x}^{2} d x\right)+\left(\int \mu(\rho) u_{x}^{2} d x\right)^{2}\right] \\
& +2 \gamma \int \rho^{\gamma} u_{x}^{2} d x+2 \gamma \int \rho^{\gamma-1} \rho_{x} u u_{x} d x-\int \mu^{\prime}(\rho) \rho_{x} u u_{x}^{2} d x \\
& +\int \mu^{\prime}(\rho) \rho u_{x}^{3} d x-\int v^{\prime}(\rho) \rho_{x} u b_{x}^{2} d x-\int v^{\prime}(\rho) \rho u_{x} b_{x}^{2} d x \\
& \leq 2 \frac{d}{d t} \int \rho^{\gamma} u_{x} d x+\frac{d}{d t} \int b^{2} u_{x} d x \\
& +C\left[\left(\int v(\rho) b_{x}^{2} d x\right)\left(\int \mu(\rho) u_{x}^{2} d x\right)+\left(\int \mu(\rho) u_{x}^{2} d x\right)^{2}\right] \\
& +C\left\|\mu(\rho) u_{x}\right\|_{L^{\infty}}\left(\int \mu(\rho) u_{x}^{2} d x+\int v(\rho) b_{x}^{2} d x\right) \\
& +2 \gamma \int \rho^{\gamma-1} \rho_{x} u u_{x} d x-\int \mu^{\prime}(\rho) \rho_{x} u u_{x}^{2} d x-\int v^{\prime}(\rho) \rho_{x} u b_{x}^{2} d x .
\end{aligned}
$$

We will first estimate the last three terms in the right hand-side of (16). We have

$$
\begin{aligned}
2 \gamma \int \rho^{\gamma-1} \rho_{x} u u_{x} d x \\
=2 \gamma \int \frac{\rho^{\gamma-1}}{\mu(\rho)} \rho_{x} u\left[\mu(\rho) u_{x}\right] d x \\
=2 \gamma \int \frac{\rho^{\gamma-1}}{\mu(\rho)} \rho_{x} u\left[\mu(\rho) u_{x}-\rho^{\gamma}\right] d x+2 \gamma \int \frac{\rho^{2 \gamma-1}}{\mu(\rho)} \rho_{x} u d x \\
=2 \gamma \int\left(\int_{0}^{\rho} \frac{\xi^{\gamma-1}}{\mu(\xi)} d \xi\right)_{x} u\left[\mu(\rho) u_{x}-\rho^{\gamma}\right] d x+2 \gamma \int\left(\int_{0}^{\rho} \frac{\xi^{2 \gamma-1}}{\mu(\xi)} d \xi\right)_{x} u d x \\
=-2 \gamma \int\left(\int_{0}^{\rho} \frac{\xi^{\gamma-1}}{\mu(\xi)} d \xi\right) u_{x}\left[\mu(\rho) u_{x}-\rho^{\gamma}\right] d x \\
\quad-2 \gamma \int\left(\int_{0}^{\rho} \frac{\xi^{\gamma-1}}{\mu(\xi)} d \xi\right) u\left[\mu(\rho) u_{x}-\rho^{\gamma}\right]_{x} d x-2 \gamma \int\left(\int_{0}^{\rho} \frac{\xi^{2 \gamma-1}}{\mu(\xi)} d \xi\right) u_{x} d x \\
\leq C \int \mu(\rho) u_{x}^{2} d x+C+C \int|u|\left|\rho u_{t}+\rho u u_{x}+\frac{1}{2} b^{2}\right| d x \\
\leq C \int \mu(\rho) u_{x}^{2} d x+C+\frac{1}{8} \int \rho u_{t}^{2} d x+C\left(\int \mu(\rho) u_{x}^{2} d x\right)^{2} .
\end{aligned}
$$

Similarly,

$$
\begin{aligned}
- & \int \mu^{\prime}(\rho) \rho_{x} u u_{x}^{2} d x \\
= & -\int \frac{\mu^{\prime}(\rho)}{\mu^{2}(\rho)} \rho_{x} u\left[\mu(\rho) u_{x}\right]^{2} d x \\
= & -\int \frac{\mu^{\prime}(\rho)}{\mu^{2}(\rho)} \rho_{x} u\left[\mu(\rho) u_{x}-\rho^{\gamma}\right]^{2} d x+\int \frac{\mu^{\prime}(\rho)}{\mu^{2}(\rho)} \rho_{x} u \rho^{2 \gamma} d x-2 \int \frac{\mu^{\prime}(\rho) \rho^{\gamma}}{\mu(\rho)} \rho_{x} u u_{x} d x
\end{aligned}
$$


Si et al. Boundary Value Problems ( 2015) 2015:62

Page 8 of 18

$$
\begin{aligned}
& =\int\left(\int_{0}^{\rho} \frac{1}{\mu(\xi)} d \xi\right)_{x} u\left[\mu(\rho) u_{x}-\rho^{\gamma}\right]^{2} d x-\int\left(\int_{0}^{\rho} \frac{\mu^{\prime}(\xi) \xi^{2 \gamma}}{\mu^{2}(\xi)} d \xi\right)_{x} u d x \\
& -2 \int\left(\int_{0}^{\rho} \frac{\mu^{\prime}(\xi) \xi^{\gamma}}{\mu^{2}(\xi)} d \xi\right)_{x} u\left[\mu(\rho) u_{x}-\rho^{\gamma}\right] d x \\
& =-\int\left(\int_{0}^{\rho} \frac{1}{\mu(\xi)} d \xi\right) u_{x}\left[\mu(\rho) u_{x}-\rho^{\gamma}\right]^{2} d x \\
& -2 \int\left(\int_{0}^{\rho} \frac{1}{\mu(\xi)} d \xi\right) u\left[\mu(\rho) u_{x} d x-\rho^{\gamma}\right]\left[\mu(\rho) u_{x}-\rho^{\gamma}\right]_{x} d x \\
& +\int\left(\int_{0}^{\rho} \frac{\mu^{\prime}(\xi) \xi^{2 \gamma}}{\mu^{2}(\xi)} d \xi\right) u_{x} d x+2 \int\left(\int_{0}^{\rho} \frac{\mu^{\prime}(\xi) \xi^{\gamma}}{\mu^{2}(\xi)} d \xi\right) u_{x}\left[\mu(\rho) u_{x}-\rho^{\gamma}\right] d x \\
& +2 \int\left(\int_{0}^{\rho} \frac{\mu^{\prime}(\xi) \xi^{\nu}}{\mu^{2}(\xi)} d \xi\right) u\left[\mu(\rho) u_{x}-\rho^{\gamma}\right]_{x} d x \\
& \leq C\left\|\mu(\rho) u_{x}\right\|_{L^{\infty}} \int \mu(\rho) u_{x}^{2} d x+C \int \mu(\rho) u_{x}^{2} d x+C \\
& +2 \int\left(\int_{0}^{\rho} \frac{\mu^{\prime}(\xi) \xi^{\gamma}}{\mu^{2}(\xi)} d \xi\right) u\left(\rho u_{t} d x+\rho u u_{x}+\frac{b^{2}}{2}\right) d x \\
& -2 \int\left(\int_{0}^{\rho} \frac{1}{\mu(\xi)} d \xi\right) u\left[\mu(\rho) u_{x}-\rho^{\gamma}\right]\left(\rho u_{t}+\rho u u_{x}+\frac{b^{2}}{2}\right) d x \\
& \leq C\left\|\mu(\rho) u_{x}\right\|_{L^{\infty}} \int \mu(\rho) u_{x}^{2} d x+C \int \mu(\rho) u_{x}^{2} d x+C \\
& +C\left(\int \mu(\rho) u_{x}^{2} d x\right)^{2}+C \int v(\rho) b_{x}^{2} d x \text {. }
\end{aligned}
$$

Similarly,

$$
\begin{aligned}
- & v^{\prime}(\rho) \rho_{x} b_{x}^{2} d x \\
= & -\int \frac{v^{\prime}(\rho) \rho_{x} u}{v^{2}(\rho)}\left[v(\rho) b_{x}\right]^{2} d x \\
= & -\int\left(\int_{0}^{\rho} \frac{v^{\prime}(\xi)}{v^{2}(\xi)} d \xi\right)_{x} u\left[v(\rho) b_{x}\right]^{2} d x \\
= & \int\left(\int_{0}^{\rho} \frac{v^{\prime}(\xi)}{v^{2}(\xi)} d \xi\right) u_{x}\left[v(\rho) b_{x}\right]^{2} d x+2 \int\left(\int_{0}^{\rho} \frac{v^{\prime}(\xi)}{v^{2}(\xi)} d \xi\right) u v(\rho) b_{x}\left[v(\rho) b_{x}\right]_{x} d x \\
= & \int\left(\int_{0}^{\rho} \frac{v^{\prime}(\xi)}{v^{2}(\xi)} d \xi\right) u_{x}\left[v(\rho) b_{x}\right]^{2} d x+2 \int\left(\int_{0}^{\rho} \frac{v^{\prime}(\xi)}{v^{2}(\xi)} d \xi\right) u v(\rho) b_{x}\left[b_{t}+(b u)_{x}\right] d x \\
\leq & C\left\|\mu(\rho) u_{x}\right\|_{L^{\infty}} \int v(\rho) b_{x}^{2} d x+\frac{1}{4} \int b_{t}^{2} d x+C\left(\int \mu(\rho) u_{x}^{2} d x\right)\left(\int v(\rho) b_{x}^{2} d x\right) \\
& +C\left(\int \mu(\rho) u_{x}^{2} d x\right)^{2}+C\left(\int v(\rho) b_{x}^{2} d x\right)^{2} \cdot
\end{aligned}
$$

Substituting all the above estimates into (16), we obtain

$$
\begin{aligned}
& \int\left(\rho u_{t}^{2}+b_{t}^{2}\right) d x+\frac{d}{d t} \int\left[\mu(\rho) u_{x}^{2}+v(\rho) b_{x}^{2}\right] d x \\
& \leq C \frac{d}{d t} \int\left(\rho^{\gamma} u_{x}+b^{2} u_{x}\right) d x+C\left(\int \mu(\rho) u_{x}^{2} d x\right)\left(\int v(\rho) b_{x}^{2} d x\right)
\end{aligned}
$$




$$
\begin{aligned}
& +C\left(\int \mu(\rho) u_{x}^{2} d x\right)^{2}+C\left\|\mu(\rho) u_{x}\right\|_{L^{\infty}} \int\left[v(\rho) b_{x}^{2}+\mu(\rho) u_{x}^{2}\right] d x \\
& +C \int \mu(\rho) u_{x}^{2} d x+C \int v(\rho) b_{x}^{2} d x+C\left(\int v(\rho) b_{x}^{2} d x\right)^{2}+C .
\end{aligned}
$$

Now, we focus on the estimate of $\left\|\mu(\rho) u_{x}\right\|_{L^{\infty}}$. Due to (7), $W^{1,1} \hookrightarrow L^{\infty}$, and Cauchy's inequality, we conclude

$$
\begin{aligned}
\left\|\mu(\rho) u_{x}\right\|_{L^{\infty}} & \leq\left\|\mu(\rho) u_{x}-\rho^{\gamma}\right\|_{L^{\infty}}+C \\
& \leq C \int\left\{\left|\mu(\rho) u_{x}-\rho^{\gamma}\right|+\left|\left[\mu(\rho) u_{x}-\rho^{\gamma}\right]_{x}\right|\right\} d x+C \\
& \leq C \int \mu(\rho)\left|u_{x}\right| d x+C \int\left|\rho u_{t}+\rho u u_{x}+\frac{1}{2} b b_{x}\right| d x+C \\
& \leq C \int \mu(\rho) u_{x}^{2} d x+C \int\left(\int \rho u_{t}^{2} d x\right)^{\frac{1}{2}} d x+C \int v(\rho) b_{x}^{2} d x+C .
\end{aligned}
$$

By (17), (18), and Cauchy's inequality, one obtains

$$
\begin{aligned}
& \int\left(\rho u_{t}^{2}+b_{t}^{2}\right) d x+\frac{d}{d t} \int\left[\mu(\rho) u_{x}^{2}+v(\rho) b_{x}^{2}\right] d x \\
& \leq C \frac{d}{d t} \int\left(\rho^{\gamma} u_{x}+b^{2} u_{x}\right) d x+C\left(\int\left[\mu(\rho) u_{x}^{2}+v(\rho) b_{x}^{2}\right] d x\right)^{2}+C .
\end{aligned}
$$

Integrating (19) with respect to $t$ over $(0, T)$ and using Cauchy's inequality, we have

$$
\begin{aligned}
& \int_{0}^{T} \int\left(\rho u_{t}^{2}+b_{t}^{2}\right) d x d t+\int\left[\mu(\rho) u_{x}^{2}+v(\rho) b_{x}^{2}\right] d x \\
& \quad \leq C \int\left(\rho^{\gamma} u_{x}+b^{2} u_{x}\right) d x+\int_{0}^{T}\left(\int\left[\mu(\rho) u_{x}^{2}+v(\rho) b_{x}^{2}\right] d x\right)^{2} d t+C \\
& \quad \leq \frac{1}{2} \int \mu(\rho) u_{x}^{2} d x+\frac{1}{2} \int v(\rho) b_{x}^{2} d x+\int_{0}^{T}\left(\int\left[\mu(\rho) u_{x}^{2}+v(\rho) b_{x}^{2}\right] d x\right)^{2} d t+C
\end{aligned}
$$

where we have used the following interpolation inequality in one dimension:

$$
\|b\|_{L^{4}} \leq C\|b\|_{L^{2}}^{\frac{1}{4}}\left\|b_{x}\right\|_{L^{2}}^{\frac{3}{4}} .
$$

Using Gronwall's inequality and (6), we complete the proof of Lemma 2.3.

Next, we focus on the $L^{2}$-estimates of $\rho_{t}$ and $\rho_{x}$, which are independent on time $t$.

Lemma 2.4 For any $0 \leq t \leq T$, one obtains

$$
\sup _{0 \leq t \leq T} \int\left(\rho_{t}^{2}+\rho_{x}^{2}\right) d x \leq C
$$

Proof Differentiating the first equation in (1) with respect to $x$, multiplying the resulting equation by $\rho_{x}$, then integrating this new equation over $I$ with respect to $x$ and using in- 
tegration by parts, one deduces

$$
\begin{aligned}
& \frac{1}{2} \frac{d}{d t} \int \rho_{x}^{2} d x \\
& =-\frac{3}{2} \int \rho_{x}^{2} u_{x} d x-\int \frac{\rho \rho_{x}\left[\mu(\rho) u_{x}\right]_{x}}{\mu(\rho)} d x+\int \frac{\rho \mu^{\prime}(\rho) \rho_{x}^{2} u_{x}}{\mu(\rho)} d x \\
& \leq C\left\|\mu(\rho) u_{x}\right\|_{L^{\infty}} \int \rho_{x}^{2} d x-\int \frac{\rho \rho_{x}}{\mu(\rho)}\left[\rho u_{t}+\rho u u_{x}+\left(\rho^{\gamma}\right)_{x}+\frac{1}{2}\left(b^{2}\right)_{x}\right] d x \\
& \leq C \int \rho u_{t}^{2} d x \int \rho_{x}^{2} d x+C \int \rho_{x}^{2} d x+C \int \rho u_{t}^{2} d x \\
& \quad+C\|b\|_{L^{\infty}}\left(\int \rho_{x}^{2} d x\right)^{\frac{1}{2}}\left(\int b_{x}^{2} d x\right)^{\frac{1}{2}} \\
& \leq C\left(\int \mu(\rho) u_{x}^{2} d x+1\right)\left(\int \rho_{x}^{2} d x\right)+C,
\end{aligned}
$$

where we have used (4), (5), (6), (7), (12), (18), and Cauchy's inequality. Then (21) together with Gronwall's inequality yields

$$
\sup _{0 \leq t \leq T} \int \rho_{x}^{2} d x \leq C
$$

By the first equation in (1), (12), and (22), one easily obtains

$$
\sup _{0 \leq t \leq T} \int \rho_{t}^{2} d x \leq C
$$

Thus, we complete the proof of Lemma 2.4.

From now on, we start to deduce the higher a priori estimates. First, we consider the $L^{2}$-estimates of $u_{x x}$ and $b_{x x}$.

Lemma 2.5 For any $0 \leq t \leq T$, one obtains

$$
\int_{0}^{T} \int\left(u_{x x}^{2}+b_{x x}^{2}\right) d x d t \leq C
$$

Proof From the second equation in (1), (7), (12), (18), and (20), we conclude

$$
\begin{aligned}
& \int_{0}^{T} \int u_{x x}^{2} d x d t \\
& \quad \leq C \int_{0}^{T} \int \rho u_{t}^{2} d x d t+C \int_{0}^{T}\left(\int u_{x}^{2} d x\right)^{2} d t+C \int_{0}^{T} \int \rho_{x}^{2} d x d t \\
& \quad+C \int_{0}^{T}\left(\int b_{x}^{2} d x\right)^{2} d t+C \int_{0}^{T}\left\|\mu(\rho) u_{x}\right\|_{L^{\infty}}^{2} \int \rho_{x}^{2} d x d t \\
& \quad \leq C .
\end{aligned}
$$


Similarly, we obtain

$$
\begin{aligned}
& \int_{0}^{T} \int b_{x x}^{2} d x d t \\
& \quad \leq C \int_{0}^{T} \int b_{t}^{2} d x d t+C \int_{0}^{T}\left(\int b_{x}^{2} d x\right)\left(\int u_{x}^{2} d x\right) d t \\
& \quad \leq C
\end{aligned}
$$

Combining (25) and (26), we obtain (24). This completes the proof.

Next, we deduce the $L^{2}$-estimates on $u_{x t}$ and $b_{x t}$.

Lemma 2.6 For any $0 \leq t \leq T$, one obtains

$$
\sup _{0 \leq t \leq T} \int\left(\rho u_{t}^{2}+b_{t}^{2}\right) d x+\int_{0}^{T} \int\left(u_{x t}^{2}+b_{x t}^{2}\right) d x d t \leq C .
$$

Proof Differentiating (13) with respect to $t$, multiplying the resulting equation by $b_{t}$, then integrating this new equation over $I$ with respect to $x$ and using integration by parts, we obtain

$$
\begin{aligned}
\frac{1}{2} \frac{d}{d t} \int \rho u_{t}^{2} d x+\int \mu(\rho) u_{x t}^{2} d x \\
=-2 \int \rho u u_{t} u_{x t} d x-\int \rho_{t} u u_{t} u_{x} d x-\int \rho u_{t}^{2} u_{x} d x \\
\quad+\int b b_{t} u_{x t} d x+\gamma \int \rho^{\gamma-1} \rho_{t} u_{x t} d x-\int \mu^{\prime}(\rho) \rho_{t} u_{x} u_{x t} d x
\end{aligned}
$$

Similarly, differentiating the third equation in (1) with respect to $t$, multiplying the resulting equation by $b_{t}$, then integrating this new equation over $I$ with respect to $x$ and using integration by parts, we have

$$
\begin{aligned}
& \frac{1}{2} \frac{d}{d t} \int b_{t}^{2} d x+\int v(\rho) b_{x t}^{2} d x \\
& \quad=-\frac{1}{2} \int b_{t}^{2} u_{x} d x-\int b_{x} b_{t} u_{t} d x+\frac{1}{2} \int b_{x} b_{t}^{2} d x-\int v^{\prime}(\rho) \rho_{t} b_{x} b_{x t} d x
\end{aligned}
$$

Combining (28) and (29), one deduces that

$$
\begin{aligned}
\frac{1}{2} \frac{d}{d t} & \int\left(\rho u_{t}^{2}+b_{t}^{2}\right) d x+\int\left[\mu(\rho) u_{x t}^{2}+v(\rho) b_{x t}^{2}\right] d x \\
= & -2 \int \rho u u_{t} u_{x t} d x-\int \rho_{t} u u_{t} u_{x} d x-\int \rho u_{t}^{2} u_{x} d x+\gamma \int \rho^{\gamma-1} \rho_{t} u_{x t} d x \\
& -\int \mu^{\prime}(\rho) \rho_{t} u_{x} u_{x t} d x+\int b b_{t} u_{x t} d x-\frac{1}{2} \int b_{t}^{2} u_{x} d x-\int b_{x} b_{t} u_{t} d x \\
& +\frac{1}{2} \int b_{x} b_{t}^{2} d x-\int v^{\prime}(\rho) \rho_{t} b_{x} b_{x t} d x \\
\leq & C\|\rho\|_{L^{\infty}}^{\frac{1}{2}}\|u\|_{L^{\infty}}\left\|\sqrt{\rho} u_{t}\right\|_{L^{2}}\left\|u_{x t}\right\|_{L^{2}}+C\|u\|_{L^{\infty}}\left\|u_{t}\right\|_{L^{\infty}}\left\|\rho_{t}\right\|_{L^{2}}\left\|u_{x}\right\|_{L^{2}}
\end{aligned}
$$




$$
\begin{aligned}
& +C\left\|u_{x}\right\|_{L^{\infty}}\left\|\sqrt{\rho} u_{t}\right\|_{L^{2}}^{2}+C\|\rho\|_{L^{\infty}}^{\gamma-1}\left\|\rho_{t}\right\|_{L^{2}}\left\|u_{x t}\right\|_{L^{2}} \\
& +C\left\|\mu^{\prime}(\rho)\right\|_{L^{\infty}}\left\|u_{x}\right\|_{L^{\infty}}\left\|\rho_{t}\right\|_{L^{2}}\left\|u_{x t}\right\|_{L^{2}}+C\left\|u_{x}\right\|_{L^{\infty}}\left\|b_{t}\right\|_{L^{2}}^{2} \\
& +C\|b\|_{L^{\infty}}\left\|b_{t}\right\|_{L^{2}}\left\|u_{x t}\right\|_{L^{2}}+C\left\|u_{t}\right\|_{L^{\infty}}\left\|b_{x}\right\|_{L^{2}}\left\|b_{t}\right\|_{L^{2}} \\
& +C\left\|b_{x}\right\|_{L^{\infty}}\left\|b_{t}\right\|_{L^{2}}^{2}+C\left\|v^{\prime}(\rho)\right\|\left\|_{L^{\infty}}\right\| b_{x}\left\|_{L^{\infty}}\right\| \rho_{t}\left\|_{L^{2}}\right\| b_{x t} \|_{L^{2}} \\
& \leq C\left(1+\left\|u_{x x}\right\|_{L^{2}}^{2}+\left\|b_{x x}\right\|_{L^{2}}^{2}\right)\left(\left\|\sqrt{\rho} u_{t}\right\|_{L^{2}}^{2}+\left\|b_{t}\right\|_{L^{2}}^{2}\right) \\
& +\frac{1}{2}\left\|\sqrt{\mu(\rho)} u_{x t}\right\|_{L^{2}}^{2}+\frac{1}{2}\left\|\sqrt{v(\rho)} b_{x t}\right\|_{L^{2}}^{2}+C,
\end{aligned}
$$

where in the last inequality we have used Cauchy's inequality, Hölder's inequality, Sobolev's embedding inequality, (6), (7), (12), and (20).

It follows from (30) that

$$
\begin{aligned}
& \frac{d}{d t} \int\left(\rho u_{t}^{2}+b_{t}^{2}\right) d x+\int\left[\mu(\rho) u_{x t}^{2}+v(\rho) b_{x t}^{2}\right] d x \\
& \quad \leq C\left(1+\left\|u_{x x}\right\|_{L^{2}}^{2}+\left\|b_{x x}\right\|_{L^{2}}^{2}\right)\left(\left\|\sqrt{\rho} u_{t}\right\|_{L^{2}}^{2}+\left\|b_{t}\right\|_{L^{2}}^{2}\right)+C,
\end{aligned}
$$

which together with (24) and Gronwall's inequality yields (27). This completes the proof.

Lemma 2.7 For any $0 \leq t \leq T$, one obtains that

$$
\sup _{0 \leq t \leq T} \int\left(u_{x x}^{2}+b_{x x}^{2}\right) d x \leq C
$$

Proof Rewrite (12) as

$$
\mu(\rho) u_{x x}=\rho u_{t}+\rho u u_{x}+b b_{x}+\gamma \rho^{\gamma-1} \rho_{x}-\mu^{\prime}(\rho) \rho_{x} u_{x} .
$$

Thus, from (4) and Minkowski's inequality, we obtain

$$
\begin{aligned}
\left\|u_{x x}\right\|_{L^{2}}^{2} \leq & C\left\|\sqrt{\rho} u_{t}\right\|_{L^{2}}^{2}+C\|\rho\|_{L^{\infty}}^{2}\|u\|_{L^{\infty}}^{2}\left\|u_{x}\right\|_{L^{2}}^{2}+C\|b\|_{L^{\infty}}^{2}\left\|b_{x}\right\|_{L^{2}}^{2} \\
& +C\left\|u_{x}\right\|_{L^{\infty}}^{2}\left\|\mu^{\prime}(\rho)\right\|_{L^{\infty}}^{2}\left\|\rho_{x}\right\|_{L^{2}}^{2}+C\|\rho\|_{L^{\infty}}^{2(\gamma-1)}\left\|\rho_{x}\right\|_{L^{2}}^{2} \\
\leq & C\left\|\sqrt{\rho} u_{t}\right\|_{L^{2}}^{2}+C\left\|u_{x}\right\|_{L^{2}}^{4}+C\left\|b_{x}\right\|_{L^{2}}^{4}+C\left\|\rho_{x}\right\|_{L^{2}}^{2} \\
& +C\left\|u_{x}\right\|_{L^{\infty}}^{2}\left\|\rho_{x}\right\|_{L^{2}}^{2}
\end{aligned}
$$

which together with (12), (20), and (27) yields

$$
\sup _{0 \leq t \leq T}\left\|u_{x x}\right\|_{L^{2}}^{2} \leq C
$$

where

$$
\sup _{0 \leq t \leq T}\left\|u_{x}\right\|_{L^{\infty}} \leq C
$$

can be shown from (12), (18), and (27). 
Similarly, rewrite the third equation in (1) as

$$
v(\rho) b_{x x}=b_{t}+b_{x} u+b u_{x}-v^{\prime}(\rho) \rho_{x} b_{x} .
$$

From (5) and Minkowski's inequality, we obtain

$$
\begin{aligned}
\left\|b_{x x}\right\|_{L^{2}}^{2} & \leq C\left\|b_{t}\right\|_{L^{2}}^{2}+C\left\|b_{x}\right\|_{L^{2}}^{2}\|u\|_{L^{\infty}}^{2}+C\|b\|_{L^{\infty}}^{2}\left\|u_{x}\right\|_{L^{2}}^{2}+C\left\|v^{\prime}(\rho)\right\|_{L^{\infty}}^{2}\left\|b_{x}\right\|_{L^{\infty}}^{2} \\
& \leq C\left\|b_{t}\right\|_{L^{2}}^{2}+C\left\|b_{x}\right\|_{L^{2}}^{2}\left\|u_{x}\right\|_{L^{2}}^{2}+C\left\|b_{x}\right\|_{L^{\infty}}^{2}\left\|\rho_{x}\right\|_{L^{2}}^{2} .
\end{aligned}
$$

By the fact that $W^{1,1} \hookrightarrow L^{\infty}$, Sobolev's embedding inequality, (5), (6), and (12), we obtain

$$
\begin{aligned}
& \left\|v(\rho) b_{x}\right\|_{L^{\infty}} \\
& \quad \leq C \int\left(\left|v(\rho) b_{x}\right|+\left|\left[v(\rho) b_{x}\right]_{x}\right|\right) d x \\
& \quad \leq C\|v(\rho)\|_{L^{\infty}}\left\|b_{x}\right\|_{L^{2}}+C\left\|b_{t}\right\|_{L^{2}}+C\left\|b_{x}\right\|_{L^{2}}\left\|u_{x}\right\|_{L^{2}} \\
& \quad \leq C,
\end{aligned}
$$

which together with (6), (12), (27), and (34) yields

$$
\sup _{0 \leq t \leq T}\left\|b_{x x}\right\|_{L^{2}}^{2} \leq C
$$

Combining (33) and (35), one can show that (31) holds. This completes the proof.

Lemma 2.8 For any $0 \leq t \leq T$, one obtains

$$
\sup _{0 \leq t \leq T} \int\left[\rho_{x x}^{2}+\rho_{x t}^{2}+\left|\left(\rho^{\gamma}\right)_{x x}\right|^{2}+\left|\left(\rho^{\gamma}\right)_{x t}\right|^{2}\right] d x+\int_{0}^{T} \int\left[\rho_{t t}^{2}+\left|\left(\rho^{\gamma}\right)_{t t}\right|^{2}\right] d x d t \leq C .
$$

Proof Differentiating the first equation in (1) twice with respect to $x$, multiplying the resulting equation by $\rho_{x x}$, then integrating this new equation over $I$ with respect to $x$ and using integration by parts, we conclude

$$
\begin{aligned}
\frac{1}{2} \frac{d}{d t} \int \rho_{x x}^{2} d x \\
=-\frac{5}{2} \int \rho_{x x}^{2} u_{x} d x-3 \int \rho_{x} \rho_{x x} u_{x x} d x-\int \rho \rho_{x x} u_{x x x} d x \\
\leq C\left\|u_{x}\right\|_{L^{\infty}} \int \rho_{x x}^{2} d x+C\left\|\rho_{x}\right\|_{L^{\infty}}\left\|\rho_{x x}\right\|_{L^{2}}\left\|u_{x x}\right\|_{L^{2}} \\
\quad+C\|\rho\|_{L^{\infty}}\left\|\rho_{x x}\right\|_{L^{2}}\left\|u_{x x x}\right\|_{L^{2}} \\
\leq C \int \rho_{x x}^{2} d x+C\left(1+\left\|\rho_{x x}\right\|_{L^{2}}\right)\left\|\rho_{x x}\right\|_{L^{2}}+C\left\|u_{x x x}\right\|_{L^{2}}^{2} \\
\leq C \int \rho_{x x}^{2} d x+C\left\|u_{x x x}\right\|_{L^{2}}^{2}+C
\end{aligned}
$$


where in the first inequality of (37) we have used Hölder's inequality and (6), and in the second inequality of (37) we have used Sobolev's inequality, Cauchy's inequality, (6), and (31).

From the first equation in (1), one can easily deduce

$$
\left(\rho^{\gamma}\right)_{t}+\left(\rho^{\gamma}\right)_{x} u+\gamma \rho^{\gamma} u_{x}=0
$$

Like (37), one obtains

$$
\frac{1}{2} \frac{d}{d t} \int\left|\left(\rho^{\gamma}\right)_{x x}\right|^{2} d x \leq C \int\left|\left(\rho^{\gamma}\right)_{x x}\right|^{2} d x+C\left\|u_{x x x}\right\|_{L^{2}}^{2}+C .
$$

Combining (37) and (39), it is easy to obtain

$$
\frac{d}{d t} \int\left[\rho_{x x}^{2}+\left|\left(\rho^{\gamma}\right)_{x x}\right|^{2}\right] d x \leq C \int\left[\rho_{x x}^{2}+\left|\left(\rho^{\gamma}\right)_{x x}\right|^{2}\right] d x+C \int u_{x x x}^{2} d x+C .
$$

Differentiating (13) with respect to $x$, we obtain

$$
\begin{aligned}
\mu(\rho) u_{x x x}= & \rho_{x} u_{t}+\rho u_{x t}+\rho_{x} u u_{x}+\rho u_{x}^{2}+\rho u u_{x x}+\left(\rho^{\gamma}\right)_{x x} \\
& +b_{x}^{2}+b b_{x x}-\mu^{\prime \prime}(\rho) \rho_{x}^{2} u_{x}-\mu^{\prime}(\rho) \rho_{x x} u_{x}-2 \mu^{\prime}(\rho) \rho_{x} u_{x x}
\end{aligned}
$$

which together with (4) gives

$$
\begin{aligned}
\int u_{x x x}^{2} d x \\
\leq C\left\|u_{t}\right\|_{L^{\infty}}^{2}\left\|\rho_{x}\right\|_{L^{2}}^{2}+C\|\rho\|_{L^{\infty}}^{2}\left\|u_{x t}\right\|_{L^{2}}^{2} \\
\quad+C\|u\|_{L^{\infty}}^{2}\left\|u_{x}\right\|_{L^{\infty}}^{2}\left\|\rho_{x}\right\|_{L^{2}}^{2}+C\|\rho\|_{L^{\infty}}^{2}\left\|u_{x}\right\|_{L^{\infty}}^{2}\left\|u_{x}\right\|_{L^{2}}^{2} \\
\quad+C\|\rho\|_{L^{\infty}}^{2}\left\|u_{x}\right\|_{L^{\infty}}^{2}\left\|u_{x x}\right\|_{L^{2}}^{2}+\left\|C\left(\rho^{\gamma}\right)_{x x}\right\|_{L^{2}}^{2} \\
\quad+C\left\|b_{x}\right\|_{L^{\infty}}^{2}\left\|b_{x}\right\|_{L^{2}}^{2}+C\|b\|_{L^{\infty}}^{2}\left\|b_{x x}\right\|_{L^{2}}^{2} \\
\quad+C\left\|\mu^{\prime \prime}(\rho)\right\|_{L^{\infty}}^{2}\left\|u_{x}\right\|_{L^{\infty}}^{2}\left\|\rho_{x}\right\|_{L^{\infty}}^{2}\left\|\rho_{x}\right\|_{L^{2}}^{2} \\
\quad+C\left\|\mu^{\prime}(\rho)\right\|_{L^{\infty}}^{2}\left\|u_{x}\right\|_{L^{\infty}}^{2}\left\|\rho_{x x}\right\|_{L^{2}}^{2}+C\left\|\mu^{\prime}(\rho)\right\|_{L^{\infty}}^{2}\left\|\rho_{x}\right\|_{L^{\infty}}^{2}\left\|u_{x x}\right\|_{L^{2}}^{2} \\
\quad C\left\|u_{x t}\right\|_{L^{2}}^{2}\left\|\rho_{x}\right\|_{L^{2}}^{2}+C\left\|u_{x t}\right\|_{L^{2}}^{2}+C\left\|u_{x}\right\|_{L^{2}}^{2}\left\|u_{x x}\right\|_{L^{2}}^{2}\left\|\rho_{x}\right\|_{L^{2}}^{2} \\
\quad+C\left\|u_{x x}\right\|_{L^{2}}^{2}\left\|u_{x}\right\|_{L^{2}}^{2}+C\left\|u_{x}\right\|_{L^{2}}^{2}\left\|u_{x x}\right\|_{L^{2}}^{2} \\
\quad+C\left\|\left(\rho^{\gamma}\right)_{x x}\right\|_{L^{2}}^{2}+C\left\|b_{x x}\right\|_{L^{2}}^{2}\left\|b_{x}\right\|_{L^{2}}^{2}+C\left\|u_{x x}\right\|_{L^{2}}^{2}\left\|\rho_{x}\right\|_{L^{2}}^{2}\left\|\rho_{x x}\right\|_{L^{2}}^{2} \\
\quad+C\left\|u_{x x}\right\|_{L^{2}}^{2}\left\|\rho_{x x}\right\|_{L^{2}}^{2}+C\left\|\rho_{x x}\right\|_{L^{2}}^{2}\left\|u_{x x}\right\|_{L^{2}}^{2} \\
\leq C\left\|u_{x t}\right\|_{L^{2}}^{2}+C\left\|\left(\rho^{\gamma}\right)_{x x}\right\|_{L^{2}}^{2}+C\left\|\rho_{x x}\right\|_{L^{2}}^{2}+C .
\end{aligned}
$$

Combining (40) and (42), we have

$$
\frac{d}{d t} \int\left[\rho_{x x}^{2}+\left|\left(\rho^{\gamma}\right)_{x x}\right|^{2}\right] d x \leq C\left[\left\|\rho_{x x}\right\|_{L^{2}}^{2}+\left\|\left(\rho^{\gamma}\right)_{x x}\right\|_{L^{2}}^{2}\right]+C\left\|u_{x t}\right\|_{L^{2}}^{2}+C
$$


which together with (27) and Gronwall's inequality yields

$$
\sup _{0 \leq t \leq T} \int\left[\rho_{x x}^{2}+\left|\left(\rho^{\gamma}\right)_{x x}\right|^{2}\right] d x \leq C
$$

Then, from the first equation in (1), (38), (7), (12), (20), (27), (31), and (43), we obtain

$$
\sup _{0 \leq t \leq T} \int\left[\rho_{x x}^{2}+\left|\left(\rho^{\gamma}\right)_{x x}\right|^{2}\right] d x+\int_{0}^{T} \int\left[\rho_{t t}^{2}+\left|\left(\rho^{\gamma}\right)_{t t}\right|^{2}\right] d x d t \leq C .
$$

Combining (43) and (44), we can show that (36) holds. This completes the proof.

Remark 2.1 It follows from (27), (36), and (41) that

$$
\int_{0}^{T} \int u_{x x x}^{2} d x d t \leq C
$$

Lemma 2.9 For any $0 \leq t \leq T$, one obtains

$$
\sup _{0 \leq t \leq T} \int\left(u_{x t}^{2}+b_{x t}^{2}\right) d x+\int_{0}^{T} \int\left(\rho u_{t t}^{2}+b_{t t}^{2}\right) d x d t \leq C .
$$

Proof From (41), we have

$$
\rho u_{t t}+\rho_{t} u_{t}+\rho_{t} u u_{x}+\rho u_{t} u_{x}+\rho u u_{x t}+\left(\rho^{\gamma}\right)_{x t}+\frac{1}{2}\left(b^{2}\right)_{x t}=\left[\mu(\rho) u_{x}\right]_{x t} .
$$

Multiplying (47) by $u_{t t}$, integrating the resulting equation over $I$ with respect to $x$, using integration by parts, and from (4) and Cauchy's inequality, one obtains

$$
\begin{aligned}
& \frac{1}{2} \frac{d}{d t} \int \mu(\rho) u_{x t}^{2} d x+\int \rho u_{t t}^{2} d x \\
&=\frac{1}{2} \int \mu^{\prime}(\rho) \rho_{t} u_{x t}^{2} d x-\int \mu^{\prime}(\rho) \rho_{t} u_{x} u_{x t t} d x-\int \rho_{t} u_{t} u_{t t} d x \\
& \quad-\int \rho_{t} u u_{x} u_{t t} d x-\int \rho u_{t} u_{x} u_{t t} d x-\int \rho u u_{x t} u_{t t} d x \\
& \quad+\left(\rho^{\gamma}\right)_{t} u_{x t t} d x+\frac{1}{2} \int\left(b^{2}\right)_{t} u_{x t t} d x \\
& \leq C\left\|\mu^{\prime}(\rho)\right\|_{L^{\infty}}\left\|\rho_{t}\right\|_{L^{\infty}} \int \mu(\rho) u_{x t}^{2} d x-\frac{d}{d t} \int \mu^{\prime}(\rho) \rho_{t} u_{x} u_{x t} d x \\
&+\int \mu^{\prime \prime}(\rho) \rho_{t}^{2} u_{x} u_{x t} d x+\int \mu^{\prime}(\rho) \rho_{t t} u_{x} u_{x t} d x+\int \mu^{\prime}(\rho) \rho_{t} u_{x t}^{2} d x \\
&-\frac{1}{2} \frac{d}{d t} \int \rho_{t} u_{t}^{2} d x+\frac{1}{2} \int \rho_{t t} u_{t}^{2} d x-\frac{d}{d t} \int \rho_{t} u u_{x} u_{t} d x \\
&+\int \rho_{t t} u u_{x} u_{t} d x+\int \rho_{t} u_{t}^{2} u_{x} d x+\int \rho_{t} u u_{x t} u_{t} d x+\frac{1}{4} \int \rho u_{t t}^{2} d x \\
&+C \int \rho u^{2} u_{x t}^{2} d x+\frac{d}{d t} \int\left(\rho^{\gamma}\right)_{t} u_{x t} d x-\int\left(\rho^{\gamma}\right)_{t t} u_{x t} d x \\
&+\frac{1}{2} \frac{d}{d t} \int\left(b^{2}\right)_{t} u_{x t} d x-\frac{1}{2} \int\left(b^{2}\right)_{t t} u_{x t} d x
\end{aligned}
$$


Differentiating the third equation in (1) with respect to $t$, one obtains

$$
b_{t t}+(b u)_{x t}=\left(v(\rho) b_{x}\right)_{x t}
$$

Multiplying (49) by $b_{t t}$, integrating the resulting equation with respect to $x$ over $I$, using integration by parts, and from (5) and Cauchy's inequality, one deduces

$$
\begin{aligned}
& \frac{1}{2} \frac{d}{d t} \int v(\rho) b_{x t}^{2} d x+\int \rho_{t t}^{2} d x \\
& =\frac{1}{2} \int v^{\prime}(\rho) \rho_{t} b_{x t}^{2} d x-\int v^{\prime}(\rho) \rho_{t} b_{x} b_{x t t} d x-\int\left(b_{x t} u+b_{x} u_{t}+b_{t} u_{x}+b u_{x t}\right) b_{t t} d x \\
& \leq C\left\|v^{\prime}(\rho)\right\|_{L^{\infty}}\left\|\rho_{t}\right\|_{L^{\infty}} \int v(\rho) b_{x t}^{2} d x-\frac{d}{d t} \int v^{\prime}(\rho) \rho_{t} b_{x} b_{x t} d x \\
& \quad+\int v^{\prime \prime}(\rho) \rho_{t}^{2} b_{x} b_{x t} d x+\int v^{\prime}(\rho) \rho_{t t} b_{x} b_{x t} d x+\int v^{\prime}(\rho) \rho_{t} b_{x t}^{2} d x \\
& \quad+\frac{1}{4}\left\|b_{t t}\right\|_{L^{2}}^{2}+C\|u\|_{L^{\infty}}^{2} \int v(\rho) b_{x t}^{2} d x+C\left\|u_{t}\right\|_{L^{\infty}}^{2}\left\|b_{x}\right\|_{L^{2}}^{2} \\
& \quad+C\left\|u_{x}\right\|_{L^{\infty}}^{2}\left\|b_{t}\right\|_{L^{2}}^{2}+C\|b\|_{L^{\infty}}^{2} \int \mu(\rho) u_{x t}^{2} d x
\end{aligned}
$$

Combining (48) and (50), we obtain

$$
\begin{aligned}
\frac{1}{2} \frac{d}{d t} \int\left[\mu(\rho) u_{x t}^{2}+v(\rho) b_{x t}^{2}\right] d x+\frac{1}{2} \int\left(\rho u_{t t}^{2}+b_{t t}^{2}\right) d x \\
\leq-\frac{d}{d t} \int \mu^{\prime}(\rho) \rho_{t} u_{x} u_{x t} d x-\frac{1}{2} \frac{d}{d t} \int \rho_{t} u_{t}^{2} d x-\frac{d}{d t} \int \rho_{t} u u_{x} u_{t} d x \\
\quad-\frac{d}{d t} \int v^{\prime}(\rho) \rho_{t} b_{x} b_{x t} d x+C \int \mu(\rho) u_{x t}^{2} d x+C \int v(\rho) b_{x t}^{2} d x \\
\quad+\frac{1}{2} \frac{d}{d t} \int\left(\rho^{\gamma}\right)_{t} u_{x t} d x+\frac{1}{2} \frac{d}{d t} \int\left(b^{2}\right)_{t} u_{x t} d x \\
\quad+C\left(\int \mu(\rho) u_{x t}^{2} d x\right)^{2}+C\left(\int v(\rho) b_{x t}^{2} d x\right)^{2}+C
\end{aligned}
$$

Integrating (51) over $(0, T)$ with respect to $t$, we deduce

$$
\begin{aligned}
& \frac{1}{2} \int\left[\mu(\rho) u_{x t}^{2}+v(\rho) b_{x t}^{2}\right] d x+\frac{1}{2} \int_{0}^{T} \int\left(\rho u_{t t}^{2}+b_{t t}^{2}\right) d x d t \\
& \leq C \int \mu\left(\rho_{0}\right) u_{x t}^{2}(0, x) d x+C \int v\left(\rho_{0}\right) b_{x t}^{2}(0, x) d x-\frac{1}{2} \int \rho_{t} u_{t}^{2} d x \\
& \quad-\int \rho_{t} u u_{x} u_{t} d x-\int v^{\prime}(\rho) \rho_{t} b_{x} b_{x t} d x+\int \mu^{\prime}(\rho) \rho_{t} u_{x} u_{x t}(0, x) d x \\
& \quad+\frac{1}{2} \int \rho_{t} u_{t}^{2}(0, x) d x+\int \rho_{t} u u_{x} u_{t}(0, x) d x+\int v^{\prime}(\rho) \rho_{t} b_{x} b_{x t}(0, x) d x \\
& \quad+C \int_{0}^{T}\left[\left(\int \mu(\rho) u_{x t}^{2} d x\right)^{2}+\left(\int v(\rho) b_{x t}^{2} d x\right)^{2}\right] d t+C .
\end{aligned}
$$


From the first and third equations in (1), we obtain

$$
\begin{aligned}
& \rho_{t}(0, x)=-\left(\rho_{0} u_{0}\right)_{x} \in H^{1}, \\
& b_{t}(0, x)=b_{0 x x}-\left(u_{0} b_{0}\right)_{x} \in H^{1},
\end{aligned}
$$

which together with (52) yields

$$
\begin{aligned}
& \int_{0}^{T} \int\left(\rho u_{t t}^{2}+b_{t t}^{2}\right) d x d t+\int\left[\mu(\rho) u_{x t}^{2}+v(\rho) b_{x t}^{2}\right] d x \\
& \leq C+\frac{1}{2} \int\left[\mu(\rho) u_{x t}^{2}+v(\rho) b_{x t}^{2}\right] d x \\
& \quad+C \int_{0}^{T}\left[\left(\int \mu(\rho) u_{x t}^{2} d x\right)^{2}+\left(\int v(\rho) b_{x t}^{2} d x\right)^{2}\right] d t
\end{aligned}
$$

which follows from Gronwall's inequality, (4), (5), (7), (12), (20), (27), (31), and (36), showing that (46) holds. This completes the proof of Lemma 2.9.

Remark 2.2 From the above estimates, we can also deduce that

$$
\begin{aligned}
& \sup _{0 \leq t \leq T} \int\left(u_{x x x}^{2}+b_{x x x}^{2}\right) d x \leq C, \\
& \int_{0}^{T} \int\left(u_{x x t}^{2}+b_{x x t}^{2}\right) d x d t \leq C,
\end{aligned}
$$

and

$$
\sup _{0 \leq t \leq T} \int\left[\rho_{t t}^{2}+\left|\left(\rho^{\gamma}\right)_{t t}\right|^{2}+b_{t t}^{2}\right] d x \leq C
$$

By combining all the estimates obtained above, we get sufficient $a$ priori estimates uniformly with $\delta$. Then letting $\delta \rightarrow 0^{+}$, we can extend the local classical solutions to the global ones. Since the process is standard [13] and [29], we omit the details here. Therefore, the proof of Theorem 1.1 is completed.

\section{Competing interests}

The authors declare that they have no competing interests.

\section{Authors' contributions}

All authors contributed significantly in writing this paper. All authors read and approved this final manuscript.

\section{Author details}

'School of Mathematical Sciences, Luoyang Normal University, Luoyang, 471022, P.R. China. ${ }^{2}$ School of Mathematical Sciences, Nankai University, Tianjin, 300071, P.R. China. ${ }^{3}$ School of Mathematics, Ocean University of China, Qingdao, 266100, P.R. China.

\section{Acknowledgements}

We would like to express our sincere thanks to the editor and the anonymous reviewers for their invaluable suggestions and helpful comments, which greatly improved the manuscript. This work was supported by NSFC-Union Science Foundation of Henan (No. U1304103). 


\section{References}

1. Cabannes, H: Theoretical Magnetofluiddynamics. Academic Press, New York (1970)

2. Kulikovskiy, AF, Lyubimov, GA: Magnetohydrodynamics. Addison-Wesley, Reading (1965)

3. Grad, H: Asymptotic theory of the Boltzmann equation. II. In: Laurmann, J (ed.) Rarefied Gas Dynamics, vol. 1, pp. 26-59. Academic Press, New York (1963)

4. Volpert, Al, Hudjaev, SI: On the Cauchy problem for composite systems of nonlinear differential equations. Math. USSR Sb. 16, 517-544 (1972)

5. Kawashima, S, Okada, M: Smooth global solutions for the one-dimensional equations in magnetohydrodynamics. Proc. Jpn. Acad., Ser. A, Math. Sci. 58, 384-387 (1982)

6. Liu, TP, Zeng, YN: Large-time behavior of solutions for general quasilinear hyperbolic-parabolic systems of conservation laws. Mem. Am. Math. Soc. 125, 599 (1997)

7. Ströhmer, G: About compressible viscous fluid flow in a bounded region. Pac. J. Math. 143, 359-375 (1990)

8. Fan, JS, Jiang, S, Nakamura, G: Stability of weak solutions to equations of magnetohydrodynamics with Lebesgue initial data. J. Differ. Equ. 251, 2025-2036 (2011)

9. Fan, JS, Jiang, S, Nakamura, G: Vanishing shear viscosity limit in the magnetohydrodynamic equations. Commun. Math. Phys. 270(3), 691-708 (2007)

10. Fan, JS, Huang, SX, Li, FC: Global strong solutions to the planar compressible magnetohydrodynamic equations with large initial data and vaccum. arXiv:1206.3624v3 [math.AP]

11. Li, XL, Su, N, Wang, DH: Local strong solution to the compressible magnetohydrodynamic flow with large data. J. Hyperbolic Differ. Equ. 8, 415-436 (2011)

12. Fan, JS, Yu, WH: Strong solution to the compressible magnetohydrodynamic equations with vacuum. Nonlinear Anal. Real World Appl. 10(1), 392-409 (2009)

13. Kawashima, S: Smooth global solutions for two-dimensional equations of electromagnetofluid dynamics. Jpn. J. Appl. Math. 1, 207-222 (1984)

14. Umeda, T, Kawashima, S, Shizuta, Y: On the decay of solutions to the linearized equations of electromagnetofluid dynamics. Jpn. J. Appl. Math. 1, 435-457 (1984)

15. Li, FC, Yu, HJ: Optimal decay rate of classical solutions to the compressible magnetohydrodynamic equations. Proc. R. Soc. Edinb., Sect. A 141, 109-126 (2011)

16. Hu, XP, Wang, DH: Global existence and large-time behavior of solutions to the threedimensional equations of compressible magnetohydrodynamic flows. Arch. Ration. Mech. Anal. 197, 203-238 (2010)

17. $\mathrm{Hu}, \mathrm{XP}$, Wang, DH: Global solutions to the three-dimensional full compressible magnetohydrodynamic flows. Commun. Math. Phys. 283, 255-284 (2008)

18. Ducomet, B, Feireisl, E: The equations of magnetohydrodynamics: on the interaction between matter and radiation in the evolution of gaseous stars. Commun. Math. Phys. 226, 595-629 (2006)

19. Fan, JS, Yu, WH: Global variational solutions to the compressible magnetohydrodynamic equations. Nonlinear Anal. 69(10), 3637-3660 (2008)

20. Zhang, JW, Jiang, S, Xie, F: Global weak solutions of an initial boundary value problem for screw pinches in plasma physics. Math. Models Methods Appl. Sci. 19, 833-875 (2009)

21. Suen, A, Hoff, D: Global low-energy weak solutions of the equations of three-dimensional compressible magnetohydrodynamics. Arch. Ration. Mech. Anal. 205, 27-58 (2012)

22. Liu, SQ, Yu, HB, Zhang, JW: Global weak solutions of 3D compressible MHD with discontinuous initial data and vacuum. J. Differ. Equ. 254, 229-255 (2013)

23. $\mathrm{Li}, \mathrm{HL}, \mathrm{Xu}, \mathrm{XY}$, Zhang, JW: Global classical solutions to 3D compressible magnetohydrodynamic equations with large oscillations and vacuum. SIAM J. Appl. Math. 45, 1356-1387 (2013)

24. LV, BQ, Shi, XD, Xu, XY: Global well-posedness and large time asymptotic behavior of strong solutions to the 2-D compressible magnetohydrodynamic equations with vacuum. arXiv:1402.4851v2 [math.AP]

25. Salvi, R, Straškraba, I: Global existence for viscous compressible fluids and their behavior as $t \rightarrow \infty$. J. Fac. Sci., Univ. Tokyo, Sect. 1A, Math. 40, 17-51 (1993)

26. Choe, HJ, Kim, H: Strong solutions of the Navier-Stokes equations for isentropic compressible fluids. J. Differ. Equ. 190 504-523 (2003)

27. Cho, Y, Kim, H: Existence results for viscous polytropic fluids with vacuum. J. Differ. Equ. 228, 377-411 (2006)

28. Cho, Y, Choe, HJ, Kim, H: Unique solvability of the initial boundary value problems for compressible viscous fluids. J. Math. Pures Appl. 83, 243-275 (2004)

29. Ding, SJ, Wen, HY, Zhu, CJ: Global classical large solutions to 1D compressible Navier-Stokes equations with density-dependent viscosity and vacuum. J. Differ. Equ. 251, 1696-1725 (2011) 